\title{
Dark-sector physics at Belle II
}

\section{Laurenza on behalf of the Belle II collaboration ${ }^{a, b, *}$}

${ }^{a} I N F N$,

Rome, Italy

${ }^{b}$ Roma Tre University,

Roma, Italy

E-mail: martina.laurenza@roma3.infn.it

The Belle II experiment at the asymmetric $e^{+} e^{-}$collider SuperKEKB is a substantial upgrade of the Belle experiment and aims at recording $50 \mathrm{ab}^{-1}$ of data over the next decade. During early physics operations, around $100 \mathrm{fb}^{-1}$ of data were collected. Specifically-designed low-multiplicity triggers enable a variety of searches for light dark matter and dark-sector mediators in the $\mathrm{GeV}$ mass range. This report presents the very first world-leading physics results from Belle II from searches for the invisible decays of a non-standard model vector boson $Z^{\prime}$, and visible decays of an axion-like particle; as well as near-term prospects for other dark-sector searches.

*** Particles and Nuclei International Conference - PANIC2021 ***

*** 5 - 10 September, $2021 * * *$

$* * *$ Online $* * *$

${ }^{*}$ Speaker 


\section{Introduction}

A variety of astrophysical observations suggest the existence of the Dark Matter (DM), consisting of particles that do not interact through strong or electromagnetic forces. The lack of evidence for non-standard model physics at the electroweak scale drives us to the possibility that DM and particles mediating its interaction with the Standard Model (SM) particles may have a mass at or below the GeV scale. This is called "light dark matter" scenario (LDM) where particles interact feebly with ordinary matter, through a limited number of possible "portals", depending on the mediator (vector portal, pseudo-scalar portal, scalar portal, neutrino portal). The efforts to detect dark matter in the sub-GeV mass bracket as well as the particle mediating the interactions with SM states, have been actively pursued at fixed target experiments and with high intensity, low-energy colliders, but the features that will be briefly described in the following, allow Belle II to lead in this field. In this report, I describe the published and ongoing searches for dark matter at Belle II.

\section{SuperKEKB and Belle II experiment}

Belle II [1] is a full upgrade of the Belle experiment located at the KEK laboratory (Tsukuba, Japan). It is built around the interaction region of SuperKEKB, an asymmetric $e^{+} e^{-}$collider that operates at a center-of-mass energy of $10.58 \mathrm{GeV}$, which corresponds to the $\mathrm{Y}(4 \mathrm{~S})$ resonance mass. The higher beam currents, the smaller interaction region and the usage of the large crossingangle nano-beam scheme allow SuperKEKB unprecedented instantaneous luminosities. Belle II is a fitting environment where to search for dark matter particles and mediators, thanks to its charged and neutral particle reconstruction performances and dedicated triggers (see for example the single-photon trigger)[2]. We are carrying on a large number of analyses in the dark sector field.

\section{Recent and ongoing dark sector searches at Belle II}

\section{1 $Z^{\prime}$ searches}

The $Z^{\prime}$ is a light gauge boson predicted by the $L_{\mu}-L_{\tau}$ model, a scenario where the $Z^{\prime}$ interacts only with the second and third generation of leptons. The $L_{\mu}-L_{\tau}$ model would explain some long standing experimental anomalies (see for example [3]). Two different decay topologies are currently analyzed at Belle II, visible and invisible, described in the Feynman diagram in Figure 1.

The search for an invisibly decaying $Z^{\prime}$ radiated off a muon was performed with a data set of $276 \mathrm{pb}^{-1}$. The signature is a peak in the distribution of the invariant mass of the system recoiling against two tracks identified as muons (Figure 2) and nothing else in the event. No excess over background expectation was found and an upper limit on the coupling constant $\mathrm{g}^{\prime}$ was set (Figure 3).

The search for $Z^{\prime}$ to invisible final states has been published by Belle II as its first physics paper [4] and an upgrade with a $\sim 300$-fold luminosity is in preparation. We search for two visible $Z^{\prime}$ decays, $e^{+} e^{-} \rightarrow \mu^{+} \mu^{-} Z^{\prime}\left(\rightarrow \mu^{+} \mu^{-}\right)$and $e^{+} e^{-} \rightarrow \mu^{+} \mu^{-} Z^{\prime}\left(\rightarrow \tau^{+} \tau^{-}\right)$. The search for a $Z^{\prime}$ decaying into a muon pair has already been performed by BaBar with $514 \mathrm{fb}^{-1}$, providing a $\mathrm{g}^{\prime}$ upper limit [5]. Belle II plans to apply an aggressive background suppression, to be competitive with the existing results with much less integrated luminosity. The main background is the $\mathrm{SM} \mu^{+} \mu^{-} \mu^{+} \mu^{-}$ production: this is reduced by exploiting the differences of kinematic distributions in events with 


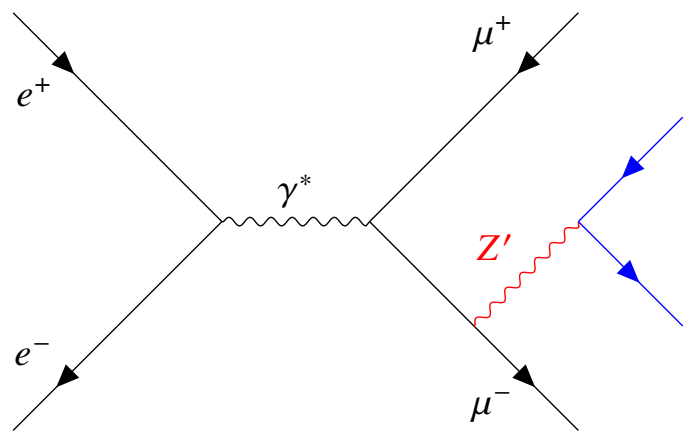

Figure 1: Feynman diagram of the process $e^{+} e^{-} \rightarrow \mu^{+} \mu^{-} Z^{\prime}$. The possible $Z^{\prime}$ products (in blue), which are currently under study at Belle II are: visible $\left(\mu^{ \pm} \mu^{\mp}, \tau^{ \pm} \tau^{\mp}\right)$ and invisible $(\nu \bar{v}, \chi \bar{\chi})$ final states.

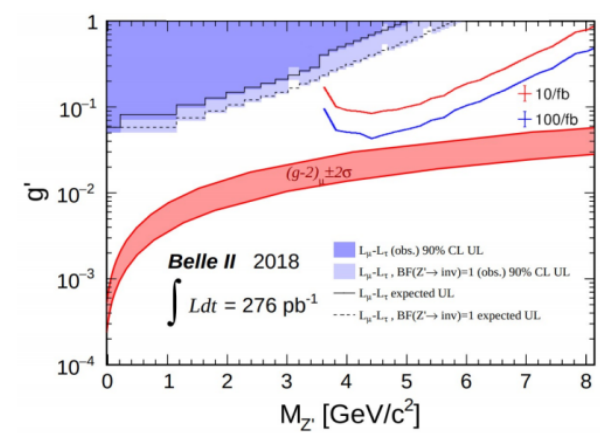

Figure 3: Expected upper limit on the coupling constant $\mathrm{g}^{\prime}$ as a function of $Z^{\prime}$ mass assuming a data set of $10 \mathrm{fb}^{-1}$ (in red) and $100 \mathrm{fb}^{-1}$ (in blue) for the $Z^{\prime} \rightarrow \tau \tau$ analysis and from the $Z^{\prime} \rightarrow$ invisible analysis of 276 $\mathrm{pb}^{-1}$ of early Belle II data (purple region).

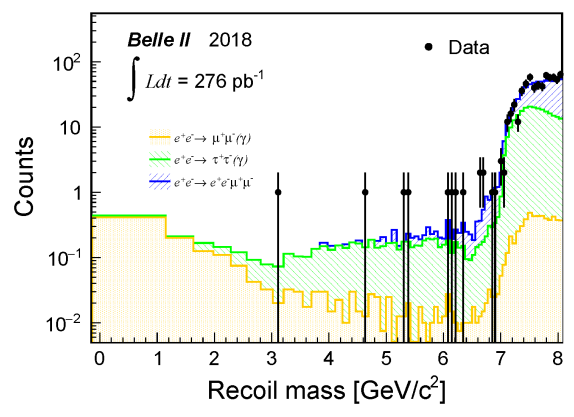

Figure 2: Recoil mass distribution for signal candidates reconstructed in $276 \mathrm{pb}^{-1}$ of early Belle II data, with simulation expectations overlaid.

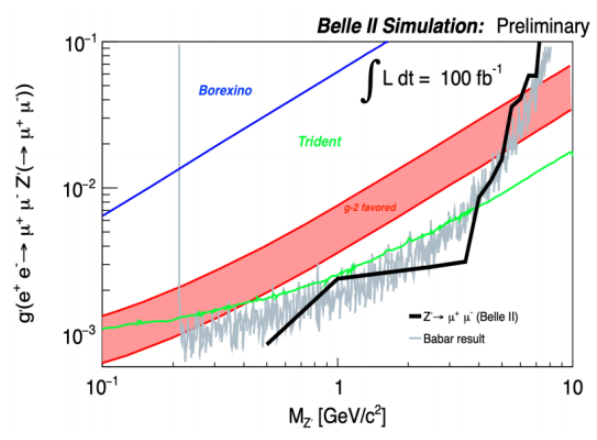

Figure 4: Expected upper limit on the coupling constant $\mathrm{g}^{\prime}$ as a function of $Z^{\prime}$ mass resulting and assuming a data set of 100 $\mathrm{fb}^{-1}$ for the $Z^{\prime} \rightarrow \mu \mu$ analysis (black line).

a $Z^{\prime} \rightarrow \mu \mu$ resonance. The actual implementation is achieved through a multi layer perceptron (MLP) based algorithm. A preliminary sensitivity study, is given in Figure 4.

The $Z^{\prime} \rightarrow \tau \tau$ aims at looking for a $Z^{\prime}$ radiated off a muon and decaying in a tau pair followed by one prong decays $(\tau \rightarrow \mathrm{e}, \mu, \pi)$. No previous searches for a $\tau \tau$ resonance in a $\mu \mu \tau \tau$ final state exist. The challenge is the presence of neutrinos. Background suppression is based on differences of kinematic distributions in events with a $Z^{\prime} \rightarrow$ resonance recoiling against a muon pair, compared with background events, and with the compatibility of the recoil system with a tau pair. The actual implementation is achieved through a MLP classifier. Main background contributions come from $\tau \tau, q \bar{q}$, ee $\mu \mu$ and $\mu \mu$ processes. A preliminary $90 \%$ CL upper limit is given in Figure 3.

\subsection{Axion-like particles search}

Axion-like particles (ALPs) are preudoscalar particles coupling to photons. Belle II aims at investigating the ALP-strahlung process. The signature is the presence of three photons summing 

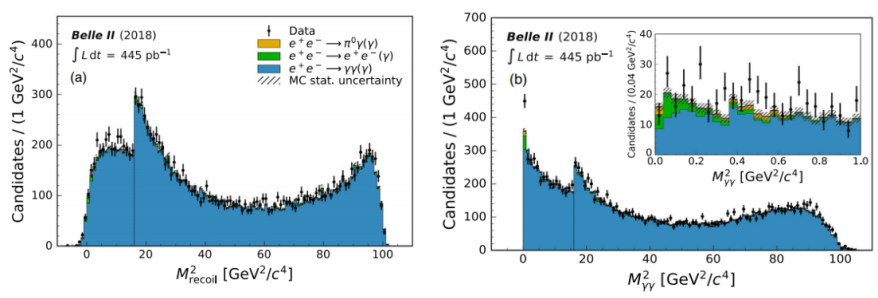

Figure 5: Squared recoil mass (on the left) and di-photon mass (on the right) distributions for signal candidates reconstructed in $445 \mathrm{pb}^{-1}$ of early Belle II data, with simulation expectations overlaid.

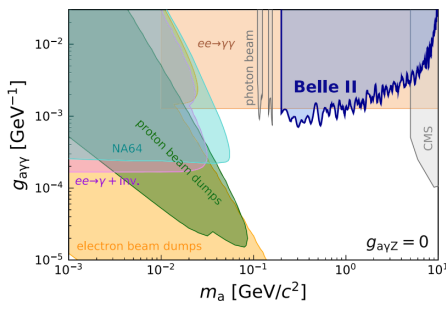

Figure 6: Upper limit on the coupling constant $\mathrm{g}^{\prime}$ for the ALP-strahlung process as a function of the recoil mass resulting analysis of $445 \mathrm{pb}^{-1}$ of early Belle II data.

up to the beam energy, no other particles, and an excess in the di-photon and in the invariant mass distributions of the system recoiling against the di-photon. (Figure 5). The main backgrounds are $e^{+} e-\rightarrow \gamma \gamma(\gamma), e^{+} e^{-}(\gamma), P \gamma \gamma$, with $P=\pi^{0}, \eta, \eta^{\prime}$. This measurement has been performed with $445 \mathrm{pb}^{-1}$, and provided $95 \%$ upper limits on the cross-section and on the coupling constant $g_{a \gamma \gamma}$ (Figure 6)[6].

\subsection{Invisible dark photon search}

The dark photon $\mathrm{A}^{\prime}$ is a massive gauge boson with spin one, that couples with the SM. Depending on the mass relationship between dark matter and the dark photon, the $\mathrm{A}^{\prime}$ can visibly decay into SM or invisibly decay into light DM. We are currently investigating the latter case. The signature for this event consists of one photon inside the calorimeter acceptance and nothing in the rest of the event and an excess in the mass (or energy) distribution of the system recoiling against the single photon. Main background sources are $e^{+} e^{-} \rightarrow \gamma \gamma \gamma, e^{+} e^{-} \gamma$ and cosmics. We expect world leading results, thanks to the calorimeter structure, with cristal gaps arranged in a non-pointing geometry, avoiding a large irreducible background of events such as $e^{+} e^{-} \rightarrow \gamma \gamma$ in which one photon escapes detection by passing through projective cracks between adjacent crystals. In Figure 7 is shown the expected upper limit on the kinetic mixing parameter $\varepsilon$.

\subsection{Dark Higgsstrahlung search}

Since the dark photon needs to be massive, one can implement, in close analogy with the SM, a spontaneous symmetry breaking mechanism of the U(1) gauge group, introducing a Higgs-like particle $\mathrm{h}^{\prime}$, called dark Higgs. The $\mathrm{h}^{\prime}$ can be produced in the Higgsstrahlung process $e^{+} e^{-} \rightarrow$ $A^{\prime *} \rightarrow h^{\prime} A^{\prime}\left(\rightarrow \mu^{+} \mu^{-}\right)$, which is also sensitive to the dark coupling constant $\alpha_{D}$. At Belle II we are investigating the case when $m_{h^{\prime}}<m_{A^{\prime}}$, with an invisible (long-lived) $\mathrm{h}^{\prime}$. The only available result comes from KLOE [7]. The signature for this event is two oppositely charged muons and missing energy and a two-dimensional peak in the distribution of the recoiling system mass versus the di-muon mass. In Figure 8 the two-dimensional distribution of the recoil mass as a function of the di-muon mass is shown. Belle II has access to unconstrained regions, which are well beyond the KLOE coverage, providing encouraging results, even with $9 \mathrm{fb}^{-1}$ only. 


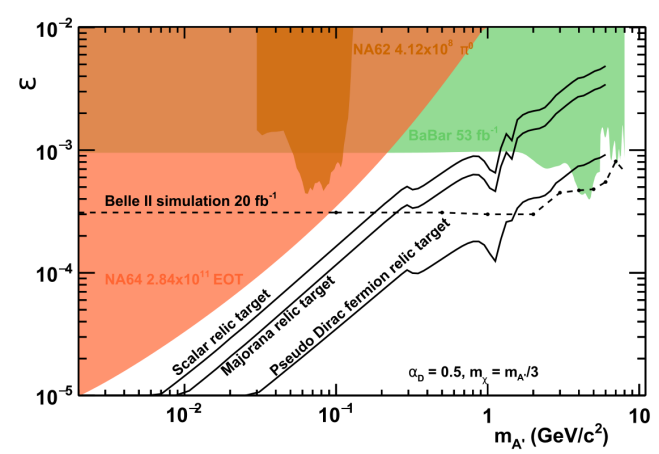

Figure 7: Expected upper limits on $\varepsilon$ for the process $e^{+} e^{-} \rightarrow \gamma A^{\prime}(\rightarrow$ invisible $)$ for $20 \mathrm{fb}^{-1}$ Belle II data set.

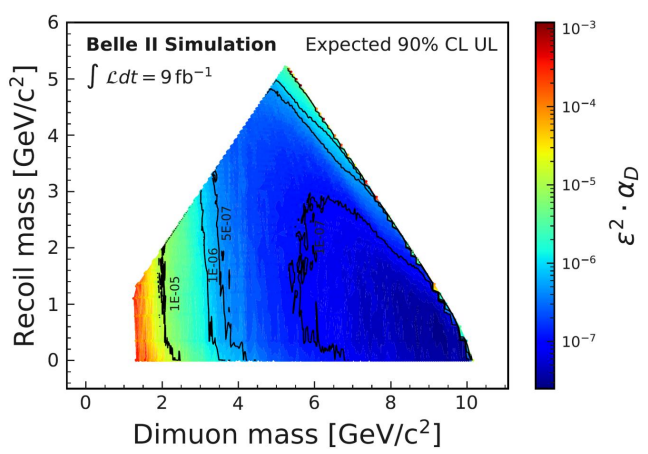

Figure 8: Two-dimension recoil vs. di-muon mass distribution in simulated data.

\section{Conclusion}

Belle II at SuperKEKB has unprecedented reach for light dark matter or mediators. It has reported already world leading results ( [4], [6]), with the luminosity collected during the 2018 pilot run $\left(500 \mathrm{pb}^{-1}\right)$. Belle II has collected $213.5 \mathrm{fb}^{-1}$ to date. A lot of dark sector searches are in progress, with world leading results expected.

\section{References}

[1] T. Abe et al., Belle II Technical Design Report, 2010.

[2] E. Kou, P. Urquijo et al., The Belle II Physics Book, Progress of Theoretical and Experimental Physic 2019 (2018) 123C01 [1808 . 10567].

[3] W. Altmannshofer, S. Gori, S. Profumo and F.S. Queiroz, Explaining dark matter and B decay anomalies with an $L_{\mu}-L_{\tau}$ model, JHEP 12 (2016) 106 [1609. 04026].

[4] I. Adachi et al., Search for an invisibly decaying $Z^{\prime}$ boson at Belle II in $e^{+} e^{-} \rightarrow \mu^{+} \mu^{-}\left(e^{ \pm} \mu^{\mp}\right)$ plus missing energy final states, 1912.11276.

[5] J. Lees, V. Poireau, V. Tisserand, E. Grauges, A. Palano, G. Eigen et al., Search for a muonic dark force at babar, Physical Review D 94 (2016) 011102.

[6] F. Abudinén, I. Adachi, H. Aihara, N. Akopov, A. Aloisio, F. Ameli et al., Search for axionlike particles produced in $e^{+} e^{-}$collisions at belle ii, Physical Review Letters 125 (2020) .

[7] A. Anastasi, D. Babusci, G. Bencivenni, M. Berlowski, C. Bloise, F. Bossi et al., Search for dark higgsstrahlung in $e^{+} e^{-} \rightarrow \mu^{+} \mu^{-}$and missing energy events with the kloe experiment, Physics Letters B 747 (2015) 365-372. 